\title{
Curcumin Protects Neonatal Rat Cardiomyocytes against High Glucose-Induced Apoptosis via PI3K/Akt Signalling Pathway
}

\author{
Wei Yu, ${ }^{1,2}$ Wenliang Zha, ${ }^{1}$ Zhiqiang Ke, ${ }^{1}$ Qing Min, ${ }^{2}$ Cairong Li, ${ }^{1}$ \\ Huirong Sun, ${ }^{3}$ and Chao Liu ${ }^{1}$ \\ ${ }^{1}$ Hubei Province Key Laboratory on Cardiovascular, Cerebrovascular, and Metabolic Disorders, \\ Hubei University of Science and Technology, Xianning 437100, China \\ ${ }^{2}$ Department of Pharmacology, Hubei University of Science and Technology, Xianning 437100, China \\ ${ }^{3}$ Department of Cardiology, Zhongnan Hospital of Wuhan University, Wuhan 430071, China \\ Correspondence should be addressed to Chao Liu; xn_liuchao@163.com
}

Received 17 July 2015; Revised 29 November 2015; Accepted 1 December 2015

Academic Editor: Yunzhou Dong

Copyright (C) 2016 Wei Yu et al. This is an open access article distributed under the Creative Commons Attribution License, which permits unrestricted use, distribution, and reproduction in any medium, provided the original work is properly cited.

\begin{abstract}
The function of curcumin on NADPH oxidase-related ROS production and cardiac apoptosis, together with the modulation of protein signalling pathways, was investigated in cardiomyocytes. Primary cultures of neonatal rat cardiomyocytes were exposed to $30 \mathrm{mmol} / \mathrm{L}$ high glucose with or without curcumin. Cell viability, apoptosis, superoxide formation, the expression of NADPH oxidase subunits, and potential regulatory molecules, Akt and GSK-3 $\beta$, were assessed in cardiomyocytes. Cardiomyocytes exposure to high glucose led to an increase in both cell apoptosis and intracellular ROS levels, which were strongly prevented by curcumin treatment $(10 \mu \mathrm{M})$. In addition, treatment with curcumin remarkably suppressed the increased activity of Racl, as well as the enhanced expression of gp $91^{\text {phox }}$ and $\mathrm{p} 47^{\text {phox }}$ induced by high glucose. Lipid peroxidation and SOD were reversed in the presence of curcumin. Furthermore, curcumin treatment markedly inhibited the reduced Bcl-2/Bax ratio elicited by high glucose exposure. Moreover, curcumin significantly increased Akt and GSK-3 $\beta$ phosphorylation in cardiomyocytes treated with high glucose. In addition, LY294002 blocked the effects of curcumin on cardiomyocytes exposure to high glucose. In conclusion, these results demonstrated that curcumin attenuated high glucose-induced cardiomyocyte apoptosis by inhibiting NADPH-mediated oxidative stress and this protective effect is most likely mediated by PI3K/Akt-related signalling pathway.
\end{abstract}

\section{Introduction}

Diabetes mellitus (DM) is becoming a global health problem that is afflicting millions of people. According to the investigation conducted by the International Diabetes Federation (IDF), the incidence of DM is rapidly increasing and the total number of people with DM will reach 592 million in 2035 [1]. Studies have indicated that diabetic people have a 2 - to 5-fold increased risk of developing heart failure [2] and that more than $50 \%-80 \%$ of diabetic patients die from diabetic cardiovascular complications [3]. Diabetic cardiomyopathy (DCM), as a major complication of DM, was initially proposed by Rubler in 1972 [4]. DCM is characterized by structural and functional cardiac disorder occurring independently of coronary artery disease and hypertension [5]. Although many research studies have attempted to elucidate its underlying mechanisms, the aetiology of DCM has never been directly determined. Numerous studies utilizing experimental animal models and clinical diabetes patients reported that diabetes enhances cardiomyocyte apoptosis not only simply in animals but also in patients $[6,7]$. Thus, cell death by apoptosis likely plays an important role in triggering the pathogenic changes in DCM [8]. Cardiomyocyte apoptosis can cause a loss of cardiac contractile muscle tissue, which eventually leads to left ventricular remodeling [9].

Both type 1 and type $2 \mathrm{DM}$ are associated with long-standing hyperglycemia. Chronic hyperglycemia has been shown to directly participate in the pathogenesis of DM-induced cardiac injury by promoting excessive oxidative stress in the heart [10], which increases cardiomyocyte apoptosis in both human and experimental DCM. Overproduction of reactive oxygen species (ROS) and a diminished antioxidant 
defence system are linked to enhanced oxidative stress in the heart in DM. Consequently, if the balance between ROS generation and ROS scavenging systems is broken, superoxide accumulates and results in cellular damage or dysfunction. Given the injurious effects of ROS in DCM, increasing attention has been placed on the administration of antioxidant agents as a compensatory therapeutic approach in DCM [11].

Curcumin, a major constituent derived from the root of Curcuma longa, has been used as a spice and food additive in India since ancient times. Today, interest in curcumin has grown rapidly due to its diverse array of biological and pharmacological activities, and it was shown to have the potential to treat inflammatory and cardiovascular diseases and cancer [12]. Curcumin has antioxidant properties that are responsible for its cardioprotective effect by enhancing antioxidant defences and eradicating ROS [13]. Additional studies have indicated that C66, a curcumin analogue, has a protective role against high glucose-induced cardiac damage via inactivation of the JNK pathway [14]. Our early stage study demonstrated that curcumin reduces cardiomyocyte remodeling and improves cardiac dysfunction by inhibiting inappropriate apoptosis in diabetic rats [15], but the mechanism through which curcumin inhibits cardiomyocyte apoptosis and oxidative stress remains unknown.

Therefore, this study was performed to determine the action of curcumin against high glucose-induced cardiac injury and elucidate the molecular mechanism of cardiomyocyte protection by exposing primary neonatal rat cardiomyocytes to a high concentration of glucose.

\section{Materials and Methods}

2.1. Animals. One- to three-day-old Sprague-Dawley rats were obtained from the experimental animal centre at Hubei University of Science and Technology. The Committee of Experimental Animals of Hubei University of Science and Technology approved this study. All animals used in this study were cared for and experimented on in accordance with the recommendations in the Guide for the Care and Use of Laboratory Animals published by the National Institutes of Health.

\subsection{Primary Culture of Neonatal Rat Cardiomyocytes and} Treatment. Neonatal rat cardiomyocytes were isolated as previously described with slight modifications [16]. The cardiomyocytes were cultured in DMEM containing $10 \%$ FBS (Gibco Life of Cells, USA), $100 \mathrm{U} / \mathrm{mL}$ penicillin, and $100 \mathrm{mg} / \mathrm{mL}$ streptomycin in a humidified air containing 5\% $\mathrm{CO}_{2}$ at $37^{\circ} \mathrm{C}$. When the cardiomyocytes reached $70 \%-80 \%$ confluence, the cells were randomized into the experimental groups: $5.5 \mathrm{mmol} / \mathrm{L} \mathrm{D}$-glucose as the normal (NG) group, $30 \mathrm{mmol} / \mathrm{L}$ D-glucose (Sigma, USA) as the high glucose (HG) group, or identical concentrations of mannitol as an osmotic control group containing $5.5 \mathrm{mmol} / \mathrm{L} \mathrm{D}$-glucose plus $24.5 \mathrm{mmol} / \mathrm{L}$ mannitol for $24 \mathrm{~h}$ in the presence or absence of curcumin $(10 \mu \mathrm{mol} / \mathrm{L})$. A subset of cardiomyocytes were exposed to LY294002 for $1 \mathrm{~h}$ before administration of high glucose and curcumin.
2.3. Assessment of Cell Viability. Cell viability was assessed with a Cell Counting Kit-8 assay kit (CCK-8, Dojindo Molecular Technologies, Japan) in 96-well plates following the instructions from the manufacturer.

2.4. Analysis of Biochemical Parameters. Lactate dehydrogenase (LDH) and aspartate amino transferase (AST) released into the culture medium as well as the malondialdehyde (MDA) level and superoxide dismutase (SOD) activity in cells were determined using the associated enzyme activity assay kits (Nanjing Jiancheng Bioengineering Research Institute, China).

2.5. Intracellular ROS Measurement. Intracellular superoxide anions were examined using the fluorescence probe dihydroethidium (DHE). Cardiomyocytes were cultured in a dark chamber at $37^{\circ} \mathrm{C}$ for $30 \mathrm{~min}$ after the application of $10 \mu \mathrm{mol} / \mathrm{L}$ DHE (Life Technology, USA) and were washed twice with PBS. Images of the cardiomyocytes were captured and analysed immediately under an inverted fluorescence microscopy (Olympus IX71, Japan).

Intracellular ROS accumulation was assessed by DCFHDA staining (Beyotime, China). Cultured cells were incubated in DMEM with $10 \mu \mathrm{mol} / \mathrm{L}$ DCFH-DA at $37^{\circ} \mathrm{C}$ for $30 \mathrm{~min}$. ROS production was detected by a Bio-Tek fluorometric imaging plate reader (excitation at $485 \mathrm{~nm}$ and emission at $528 \mathrm{~nm}$ ).

Furthermore, intracellular ROS was also measured by high performance liquid chromatography (HPLC) (SHIMADZU, LC-20AD, Japan) using a DHE fluorescent probe as previously described [17]. Briefly, cardiomyocytes were treated with DHE $(10 \mu \mathrm{mol} / \mathrm{L})$ for $30 \mathrm{~min}$ and then incubated with $0.1 \%$ Triton X-100 dissolved in PBS to permeabilize the cell membrane. Protein determination was performed using a Bicinchoninic Acid (BCA) protein assay kit (Beyotime, China). Then, $100 \mu \mathrm{L}$ of cell lysate was added to an equal volume of $0.2 \mathrm{~mol} / \mathrm{L} \mathrm{HClO}_{4}$ in methanol, and the mixture was put on ice for $2 \mathrm{~h}$ to precipitate the proteins. Afterwards, the resulting mixture was centrifuged at $20,000 \mathrm{~g}$ at $4^{\circ} \mathrm{C}$ for $30 \mathrm{~min}$. Then the supernatant was collected and neutralized with $1 \mathrm{~mol} / \mathrm{L}$ potassium phosphate buffer $(\mathrm{pH}$ 2.6). The supernatant was spun again for $15 \mathrm{~min}$ and subjected to the HPLC analysis (excitation at $490 \mathrm{~nm}$ and emission at $596 \mathrm{~nm})$.

2.6. TUNEL Assay. Apoptotic cardiomyocytes were detected using a terminal deoxynucleotidyl transferase dUTP nick end labelling (TUNEL) assay kit obtained from Roche Applied Science. Briefly, $4 \%$ paraformaldehyde and $0.1 \%$ Triton X100 were used to fix and permeabilize the cardiomyocytes on glass slides. After rinsing the cardiomyocytes with PBS, they were added and incubated with the TUNEL reagents according to the instructions from the manufacturer. Images were captured under a fluorescence microscope (Olympus BX53, Japan) and the proportion of TUNEL-positive cells was estimated using the following formula: TUNELpositive cardiomyocytes/total number of cardiomyocytes $x$ $100 \%$. 
2.7. Flow Cytometry. Flow cytometry was performed using an Annexin V-FITC Apoptosis Detection Kit (Best Bio, China) following the manufacturer's protocol. Briefly, after being treated with the appropriate drugs, the cardiomyocytes were harvested with trypsin and washed with cold PBS. Afterwards, the collected cardiomyocytes were isolated by centrifugation, resuspended in $500 \mu \mathrm{L}$ of $1 \mathrm{x}$ binding buffer, and treated with $5 \mu \mathrm{L}$ Annexin V-FITC and $5 \mu \mathrm{L}$ PI for 15 min at $4^{\circ} \mathrm{C}$ in the dark. Apoptotic cardiomyocytes were detected by flow cytometer (Becton Dickinson, USA).

2.8. Determination of Rac1 Activity. Racl activity was determined using a Racl Activation Assay Kit (Millipore, USA) following the instructions from the manufacturer. Cultured cardiomyocytes were homogenized with 1x MLB. Rac-GTP was immunoprecipitated using PAK1-PBD agarose beads coated with an anti-active Rac-GTP mouse monoclonal antibody. Finally, the extracts were analysed by western blot.

2.9. Western Blot Analysis. Cardiomyocytes were lysed with 1x RIPA lysis buffer (Cell Signalling Technology, USA). After centrifugation, the lysates were clarified, and the supernatants fractions were isolated. Protein concentrations in cells were defined by the BCA protein assay. Approximately $30-50 \mu \mathrm{g}$ of protein was loaded and separated by SDS-PAGE gels and then transferred to a PVDF membrane. After blocking the membrane with $5 \%$ nonfat milk, the following primary antibodies were used for western blot: Bcl-2, Bax, Akt, GSK$3 \beta$, phospho-Akt (Ser473), phospho-GSK-3 $\beta$ (Ser9) (Cell Signalling Technology, USA), gp91 ${ }^{\text {phox }}, \mathrm{p} 7^{\text {phox }}$, and $\beta$-actin (Santa Cruz Biotechnology, USA). Then the membrane was probed with appropriate secondary antibodies. Finally, the blots were visualized using a chemiluminescence system (Pierce Biosciences, USA). Image analysis software (GeneTools from SynGene) was used to quantify the immunoblots.

2.10. Statistics. The values are expressed as mean \pm SD from repeated experiments. Statistical analysis was performed using ANOVA, and a $P$ value of $<0.05$ was considered to indicate a significant difference for all the values.

\section{Results}

3.1. Curcumin Inhibited High Glucose-Induced Cardiomyocytes Injury. To ascertain the role of curcumin in cell survival, we examined the viability of primary cultured neonatal rat cardiomyocytes incubated with different doses of curcumin for $24 \mathrm{~h}$ using a CCK-8 assay. As presented in Figure 1(a), compared with the NG group, cell viability was markedly decreased at the high glucose concentration of $30 \mathrm{mmol} / \mathrm{L}$, and mannitol $(30 \mathrm{mmol} / \mathrm{L})$ employed as an osmotic control agent did not mimic the effects of $30 \mathrm{mmol} / \mathrm{L}$ D-glucose. In the cardiomyocytes exposed to high glucose, curcumin treatment increased cell viability in a dose-dependent manner.

$\mathrm{LDH}$ and AST are oxidoreductase enzymes that are present in the cytosol of animals and plants. They are highly stable enzymes that can be used to evaluate tissue and cell damage. As shown in Figures 1(b) and 1(c), the amounts of
LDH and AST released by the cardiomyocytes were much higher in the HG group than those in the NG group. Interestingly, the cardiomyocytes exposed to high glucose and treated with curcumin released significantly lower amounts of LDH and AST into the medium than the cardiomyocytes undergoing high glucose alone.

3.2. Curcumin Abrogated High Glucose-Induced Cardiomyocytes Apoptosis. Apoptotic cardiomyocytes were detected by both TUNEL staining and flow cytometry. The TUNEL assay showed few apoptotic cardiomyocytes in the NG group and a greater number of apoptotic cardiomyocytes in the HG group. However, cotreatment with high glucose and curcumin $(10 \mu \mathrm{M})$ abrogated the increase in TUNEL-positive cells triggered by high glucose (Figures 2(a) and 2(b)).

The flow cytometric analysis also suggested that the administration of curcumin resulted in an evident decrease in the number of apoptotic bodies compared to cardiomyocytes exposed to high glucose that were not treated with curcumin (Figures 2(c) and 2(d)).

It is well known that apoptotic-related proteins regulate the progression of apoptosis. Thus, we performed further experiments to investigate if these regulatory proteins contributed to the effect of the inhibition of high glucose-induced apoptosis by curcumin. As indicated by immunohistochemical staining (Figures 3(a) and 3(b)), in contrast to the NG group, the HG group had reduced $\mathrm{Bcl}-2$ expression and enhanced Bax expression. As we predicted, curcumin significantly enhanced $\mathrm{Bcl}-2$ expression and reduced Bax expression in cardiomyocytes exposed to high glucose. Western blot showed that the Bcl-2/Bax ratio was remarkably reduced in the HG group compared to the NG group (Figures 3(c) and $3(\mathrm{~d})$ ). After treatment with curcumin, Bcl-2 expression was elevated, Bax expression was greatly reduced, and the Bcl2/Bax ratio was significantly upregulated.

3.3. Curcumin Decreased High Glucose-Induced ROS Generation, Reduced MDA Content, and Increased SOD Activity in Cardiomyocytes. MDA formation and SOD activity, indexes of lipid superoxide, and oxygen free radical levels were measured in the cardiomyocytes. The MDA level in cardiomyocytes was significantly increased in the HG group. In contrast, SOD activity was found to be decreased when comparing the HG group with the NG group. The cardiomyocytes exposed to high glucose with curcumin markedly decreased the MDA level and enhanced SOD activity compared to those not treated with curcumin (Figures 4(a) and 4(b)).

ROS (a key executor of oxidative stress) was measured by DHE staining, DCFH-DA staining, and HPLC assay, which indicated that the ROS level in the HG group was much higher than that in the NG group. However, the HG-induced increase in ROS was strongly blocked by treatment with curcumin (Figures 4(c)-4(f)).

3.4. Curcumin Attenuated High Glucose-Induced Expression of NADPH Oxidase Isoforms in Cardiomyocytes. Because NADPH oxidase activation is directly related to increased 


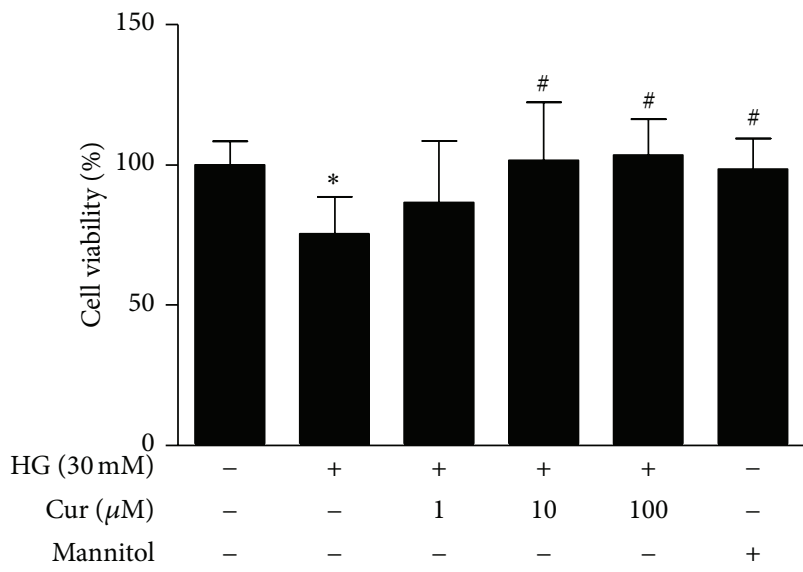

(a)

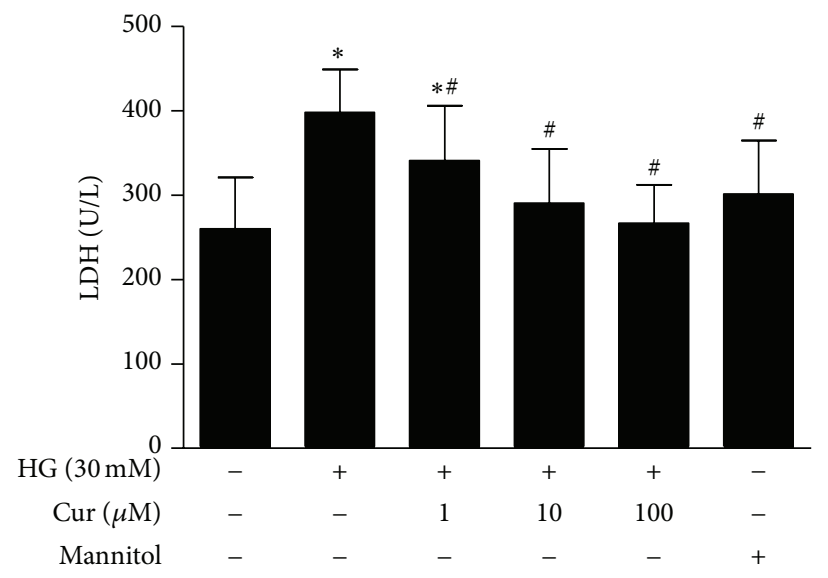

(b)

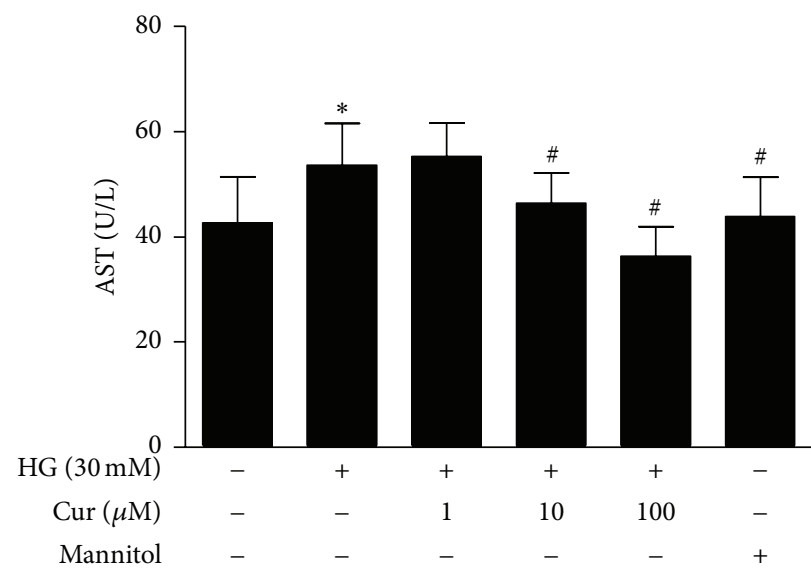

(c)

FIGURE 1: Curcumin increased cell viability and inhibited injury in cardiomyocytes exposure to high glucose. (a) Cell viability was examined with a CCK-8 assay. (b) Curcumin decreased the level of LDH in the supernatant. (c) Curcumin decreased the level of AST in the supernatant. Values are presented as mean \pm SD. ${ }^{*} P<0.05$ versus NG group. ${ }^{\#} P<0.05$ versus HG group. $n=10$.

oxidant production induced by hyperglycemia [18], we evaluated Racl activity and gp91 $1^{\text {phox }}$ and $47^{\text {phox }}$ (NADPH subunits) expression. As presented in Figure 5, Racl activity in cardiomyocytes was much higher in the HG group than in the NG group. Moreover, increased expression of gp $91^{\text {phox }}$ and $\mathrm{p} 47^{\mathrm{phox}}$ was detected in the HG group, while Racl activity and the expression of gp91 ${ }^{\text {phox }}$ and $\mathrm{p} 47^{\text {phox }}$ were markedly inhibited by curcumin in the cardiomyocytes exposed to high glucose. These data suggest that the protective role of curcumin against HG-induced cardiac injury is largely through inhibition of NADPH oxidase-mediated ROS production.

\subsection{Curcumin Activated the PI3K/Akt/GSK-3 $\beta$ Signalling} Pathway in Cardiomyocytes. The activation of the PI3K/Akt signalling pathways is well known to inhibit HG-induced apoptosis [19]. Therefore, we targeted the PI3K/Akt signalling pathway to determine the mechanism through which curcumin inhibits HG-induced apoptosis. Akt and GSK-3 $\beta$ phosphorylation were markedly decreased in the HG group as compared with the NG group. Cardiomyocytes treated with curcumin showed a remarkable increase in expression of Akt and GSK- $3 \beta$ phosphorylation. Pretreatment with the PI3K inhibitor LY294002 reversed the increased effect of curcumin on Akt and GSK-3 $\beta$ phosphorylation. In parallel with that, the expression of $\mathrm{Bcl}-2$ and Bax regulated by curcumin was abolished by treatment with LY294002 and curcumin failed to reduce gp $91^{\text {phox }}$ and $\mathrm{p} 47^{\text {phox }}$ expression levels when LY294002 was applied. These results indicate that PI3K/Akt signalling may be involved in the inhibition of apoptosis by curcumin in cardiomyocytes exposed to high glucose (Figure 6).

\section{Discussion}

DCM is frequently seen in asymptomatic diabetic patients. It is now recognized as left ventricular dysfunction associated with increasing the danger of heart failure without hypertension and coronary artery disease or valvular heart diseases [6]. DM-induced left ventricular dysfunction includes impaired systolic and diastolic function, but diastolic dysfunction can occur prior to systolic dysfunction and can be characterized as the early phase of DCM [20]. 

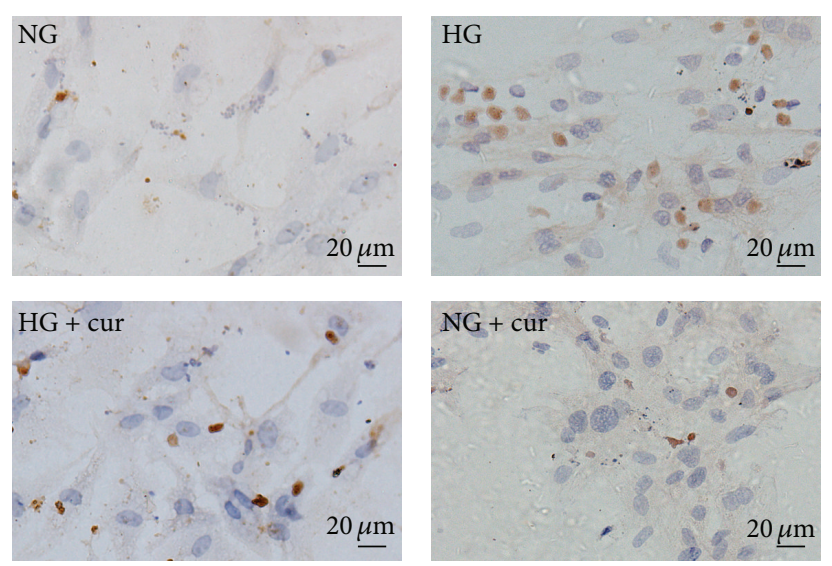

(a)
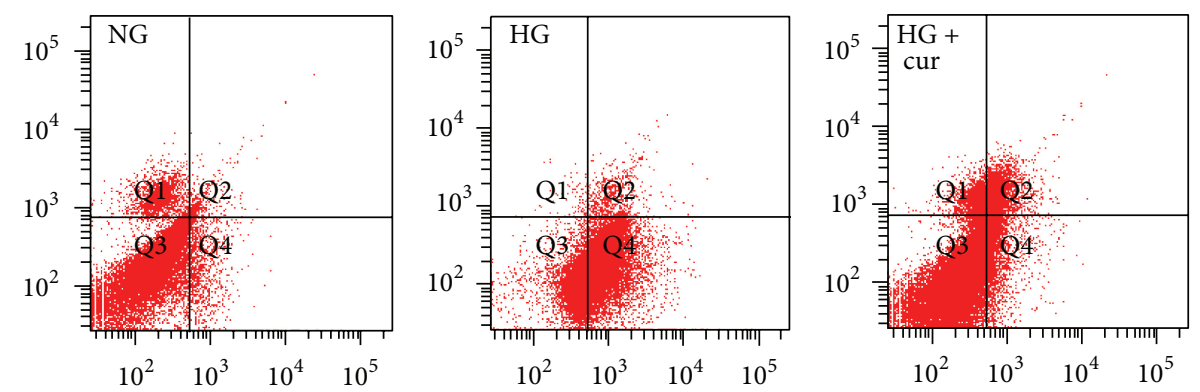

(c)

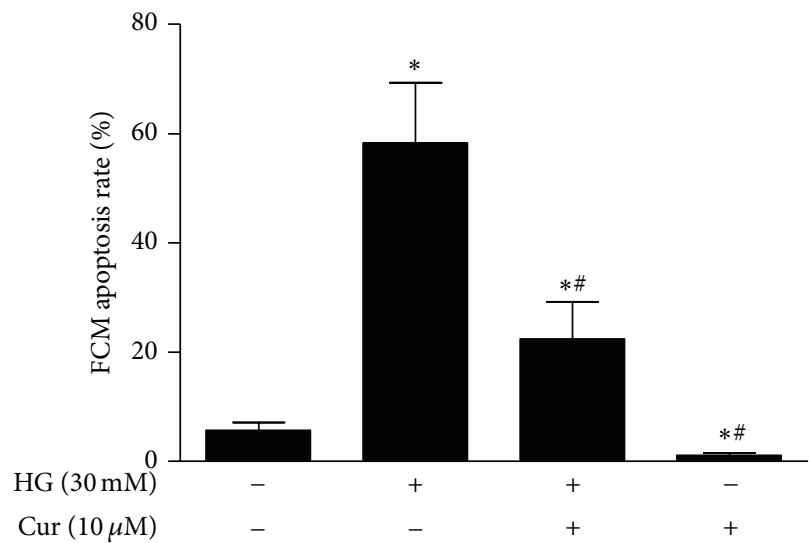

(d)

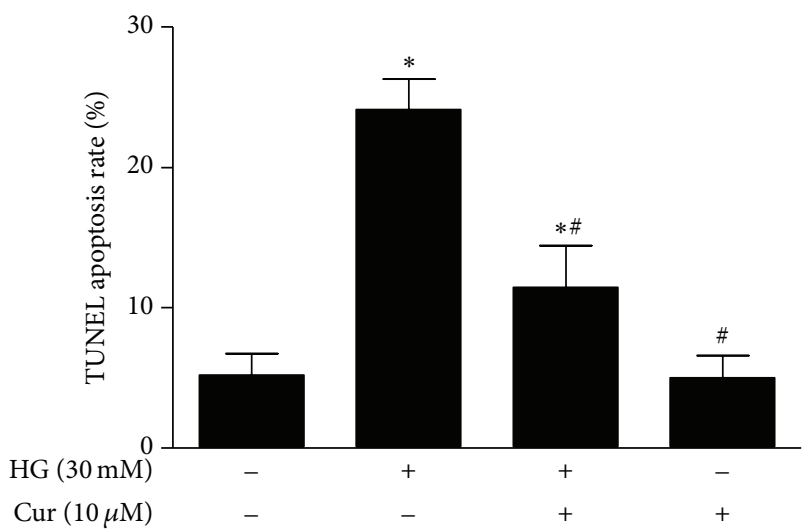

(b)

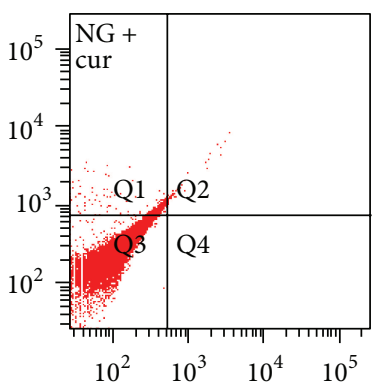

FIGURE 2: Curcumin inhibited high glucose-induced cardiomyocytes apoptosis. (a) Representative images of apoptotic cardiomyocytes stained by TUNEL (magnification $=400 \mathrm{x}$, bar is $20 \mu \mathrm{m}$ ). (b) Quantitative analysis of TUNEL staining. (c) Representative images of apoptotic cardiomyocytes observed using FCM. (d) Quantitative analysis of the FCM results. Values are presented as mean \pm SD. ${ }^{*} P<0.05$ versus NG group. ${ }^{\#} P<0.05$ versus $H G$ group.

Several studies have shown a close correlation between left ventricular diastolic dysfunction and myocardial apoptosis and have shown that cardiac function can be improved by sufficient control of myocardial apoptosis [21]. Massive loss of cardiomyocytes due to various apoptotic stimuli occurs, resulting in fibrosis and, eventually, heart failure due to the lack of cardiomyocyte proliferation greatly limiting the generation of new cardiomyocyte. In this context, a clear reduction in cardiomyocyte apoptosis is regarded as a latent therapeutic strategy for the treatment of DCM. In the current work, we found that curcumin lessened cardiomyocyte apoptosis induced by high glucose and observed a curcumin-induced reduction in Bax, which plays a crucial role in mitochondrion-mediated apoptosis by being inserted into the mitochondrial outer membrane and resulting in the release of proapoptotic factors. In contrast, Bcl-2 is 


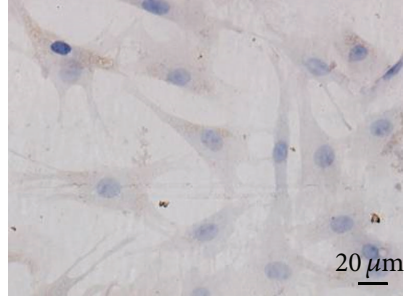

I: NG

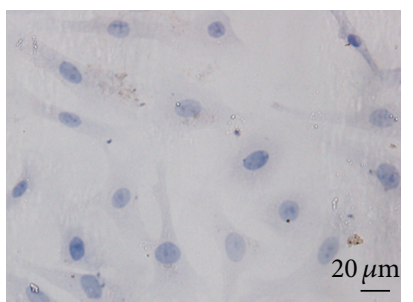

I: NG

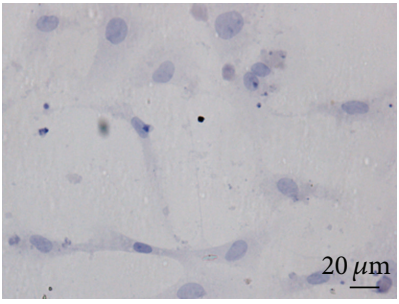

II: HG

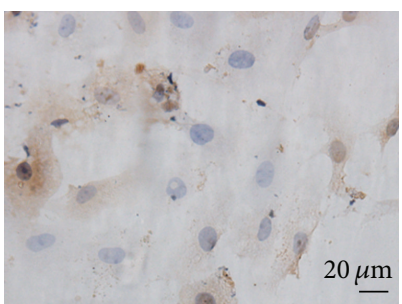

II: HG

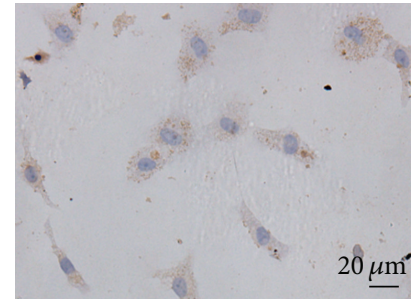

III: HG + cur

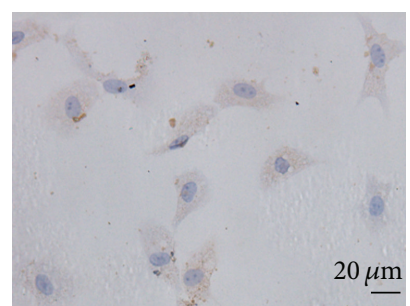

III: HG + cur

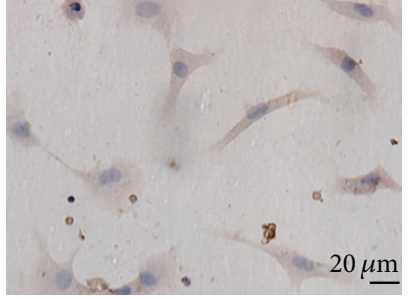

IV: $\mathrm{NG}+$ cur

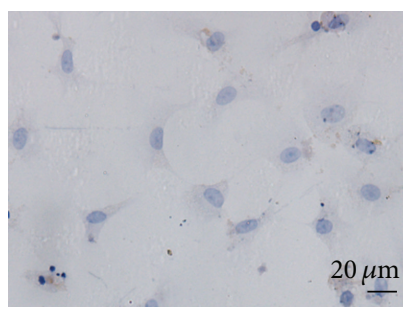

IV: NG + cur

(b)

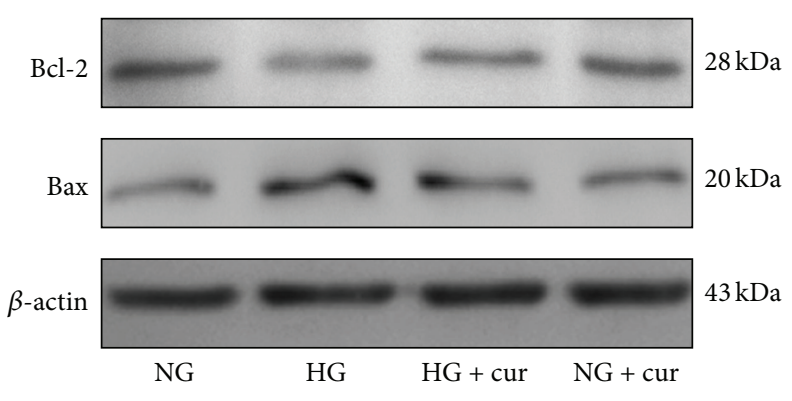

(c)

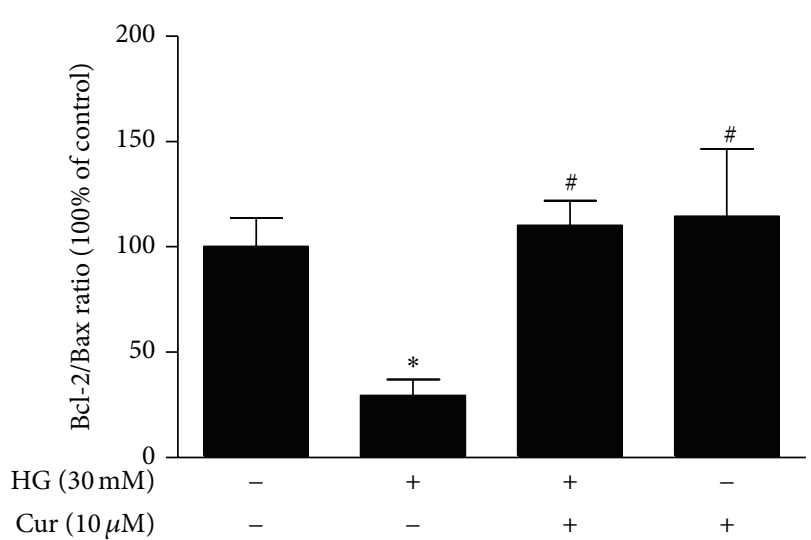

(d)

Figure 3: Curcumin regulated the expression of Bax and Bcl-2 in cardiomyocytes exposed to high glucose. (a) Representative images of Bcl-2 by immunohistochemical staining (magnification $=400 \mathrm{x}$, bar is $20 \mu \mathrm{m}$ ). (b) Representative images of Bax by immunohistochemical staining (magnification $=400 \mathrm{x}$, bar is $20 \mu \mathrm{m}$ ). (c) Representative images of Bax and Bcl-2 expression by western blot. (d) Quantitative analysis of the Bcl-2/Bax ratio. $n=3$. Values are presented as mean \pm SD. ${ }^{*} P<0.05$ versus NG group. ${ }^{\#} P<0.05$ versus HG group.

an antiapoptotic protein that prevents Bax oligomerization and was increased by curcumin treatment. Thus, curcumin's cardioprotective effects are possibly mediated by normalization of the Bcl-2/Bax ratio.

Cumulative evidence suggests that both cardiomyocyte apoptosis and oxidative stress contribute to the pathogenesis and development of diabetic cardiovascular complications [22]. Previous researches have provided direct evidence that the sustained generation of ROS during oxidative stress leads to cardiomyocyte apoptosis, which contributes to the development of DCM [23]. Emerging evidence has confirmed that activation of NADPH oxidase-generated ROS signalling is related to apoptosis in cardiomyocytes exposed to a hyperglycemic environment [24]. NADPH oxidase contains two membrane-bound subunits (gp91 ${ }^{\text {phox }}$ and $\mathrm{p} 22^{\text {phox }}$ ) and four cytosolic regulatory subunits, including $\mathrm{p} 40^{\text {phox }}, \mathrm{p} 47^{\text {phox }}$, p67 $7^{\text {phox }}$, and Racl. Racl plays a crucial role in the assembly of NADPH oxidase, which generates superoxide [25] and is a central factor in NADPH-mediated cardiomyocytes apoptosis in response to high glucose levels [26]. Therefore, the deleterious consequences of overactivation of NADPH oxidase in the form of diabetic cardiovascular complications have been well established. NADPH oxidase can impair the redox balance, thus inducing or exacerbating intracellular oxidative stress and resulting in abnormal ROS production. Therefore, inhibition of excessive ROS produced by NADPH oxidase appears to be another possible target for preventing the development of DCM [27]. Consistent with previous studies, we also found that lipid peroxidation levels were enhanced when the cardiomyocytes were exposed to high glucose, which was accompanied by an elevation in ROS generation as a result of the activation of NADPH 


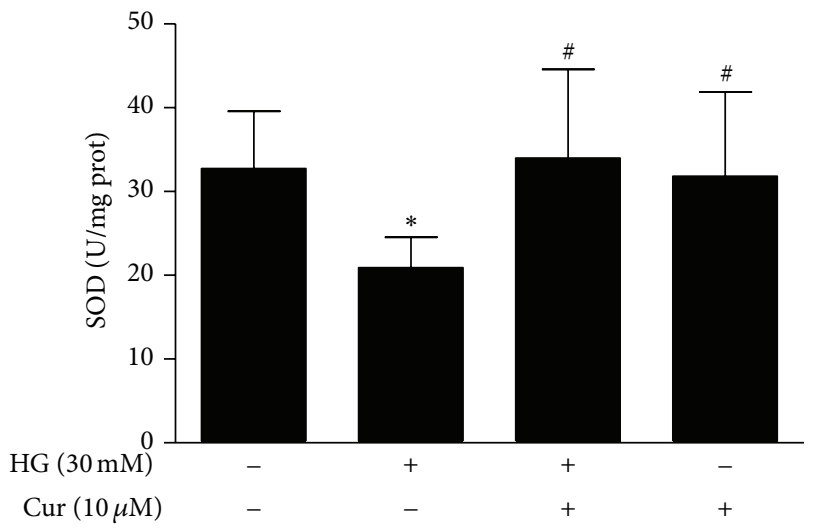

(a)
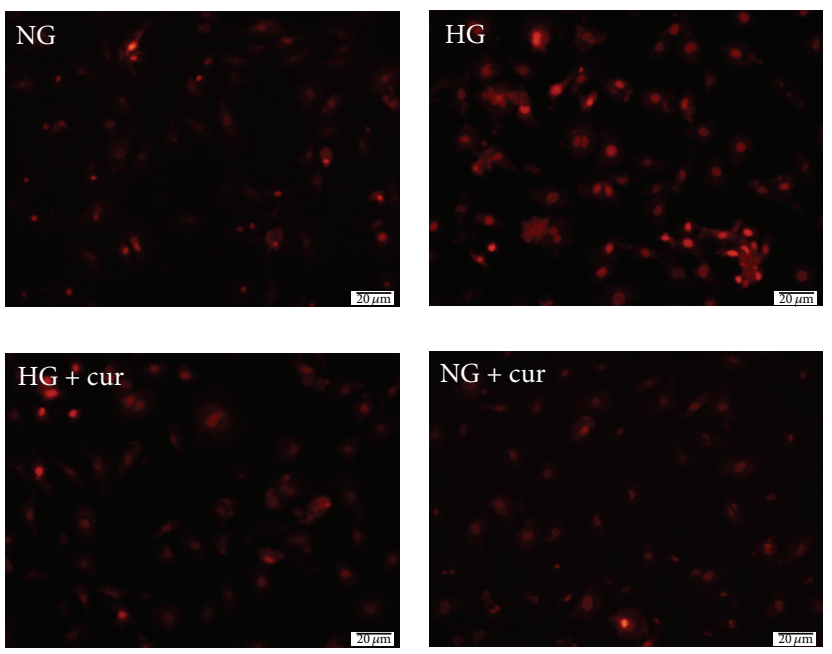

(c)

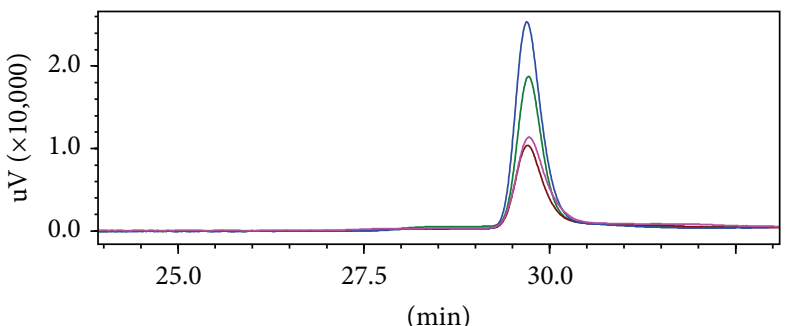

- NG

— HG + cur

_ HG

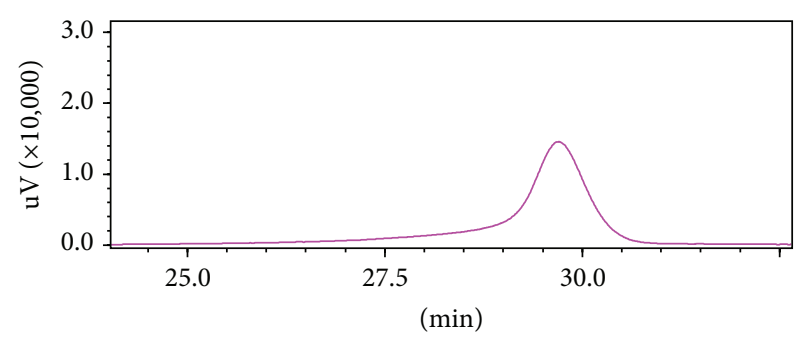

(e)

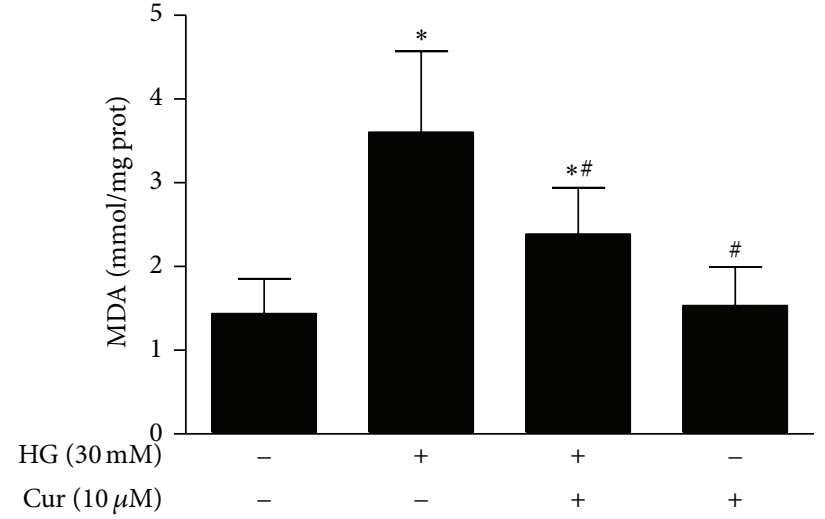

(b)

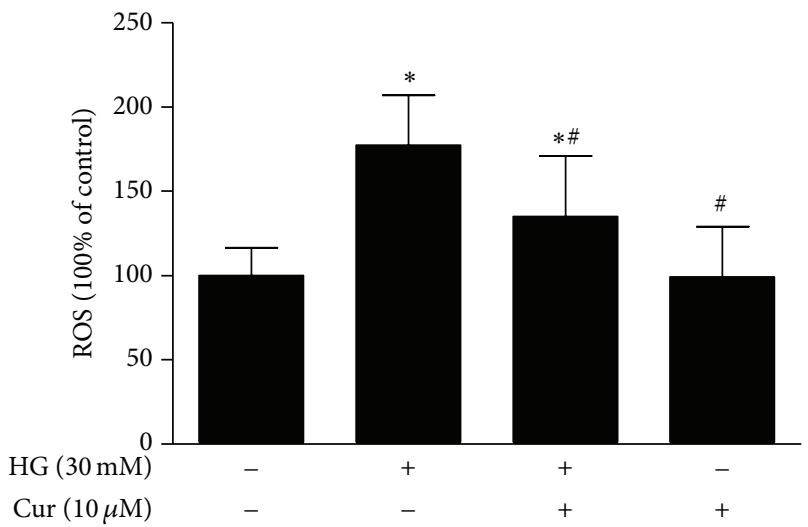

(d)

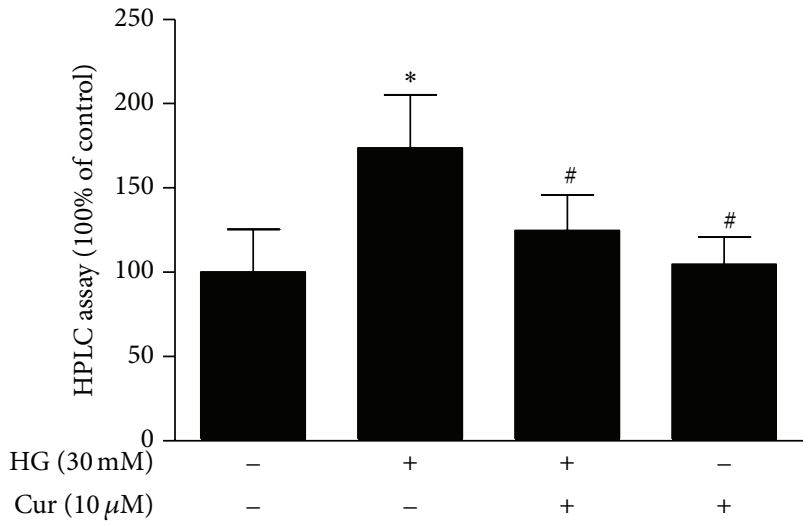

(f)

FIGURE 4: Curcumin suppressed high glucose-induced cardiomyocytes oxidative stress. (a) Curcumin enhanced SOD activity in cardiomyocytes $(n=12)$. (b) Curcumin reduced MDA level in cardiomyocytes $(n=10)$. (c) Representative images of DHE staining $(n=3)$. (d) Quantification of DCFH-DA staining $(n=11-12)$. (e) Representative images of HPLC assay $(n=4-6)$. (f) Quantification of HPLC assay. Values are presented as mean \pm SD. ${ }^{*} P<0.05$ versus NG group. ${ }^{\#} P<0.05$ versus $H G$ group. 


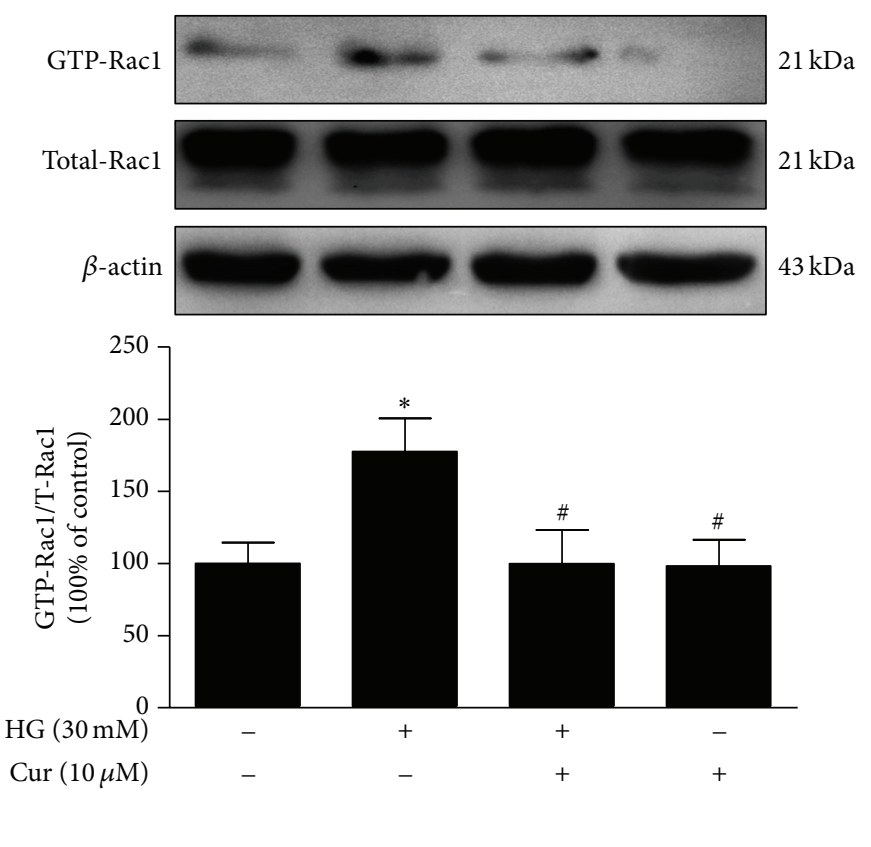

(a)

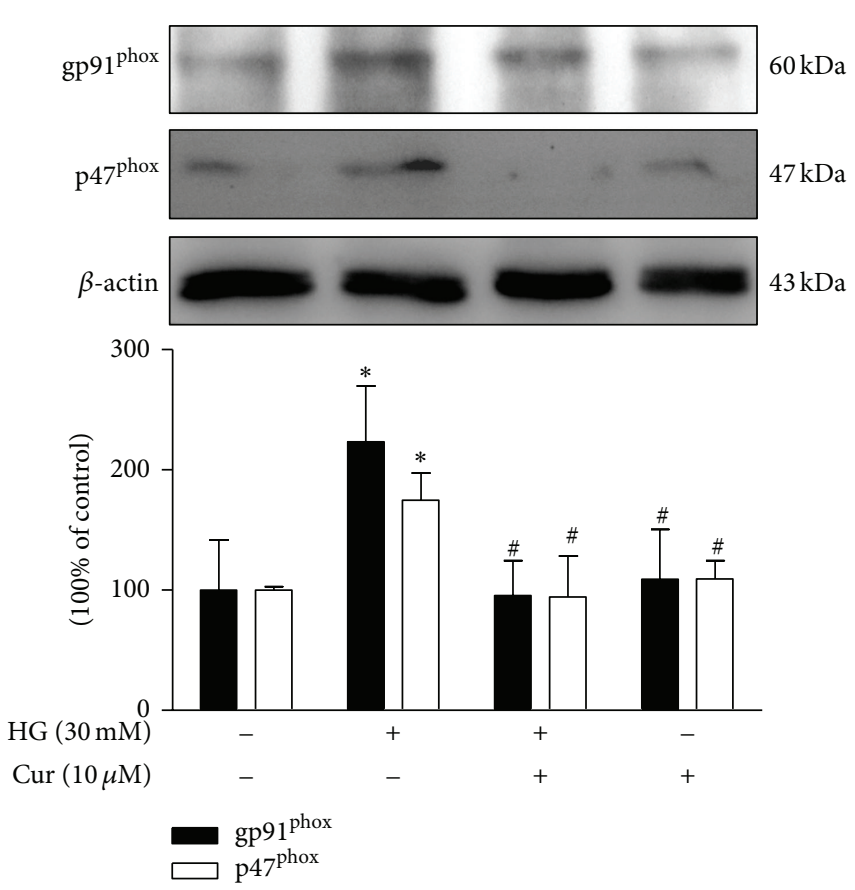

(b)

FIGURE 5: Curcumin decreased high glucose-induced Racl activity and the expression of gp91 ${ }^{\text {phox }}$ and p47 ${ }^{\text {phox }}$. (a) Racl activity assay. (b) Western blots analysis of gp91 ${ }^{\text {phox }}$ and $\mathrm{p} 47^{\text {phox }}$ expression. $n=3$. Values are presented as mean $\pm \mathrm{SD} .{ }^{*} P<0.05$ versus NG group. ${ }^{\#} P<0.05$ versus HG group.

oxidase. High glucose triggers NADPH oxidase activation by improving Racl activation and enhancing gp91 ${ }^{\text {phox }}$ and gp $47^{\text {phox }}$ expression. In agreement with earlier studies that showed that curcumin has a multitude of cardioprotective effects attributed to its efficient antioxidant capacity, we found that treatment with curcumin suppressed a hyperglycemiainduced rise in ROS generation through inactivation of NADPH oxidase.

Impaired Akt/GSK-3 $\beta$ signalling pathway has been shown to be involved in the development of metabolic disorders. Akt is responsible for the modulation of cardiovascular functions linked with cardiac growth and survival, contractile function, and coronary angiogenesis [28]. In particular, Akt1 has been demonstrated to play an antagonist role against pathological cardiac hypertrophy, which is an inevitable precursor of heart failure. Consistent with these observations, $\mathrm{Aktl}^{-/-}$mice displayed multiple heart defects, including enhanced cardiac growth and insufficient cardiomyocyte contractility [29]. Furthermore, Akt2 exerts a particularly important impact on the regulation of glucose metabolism and supports cell survival by restraining apoptosis via activation or inactivation of a number of target proteins involved in the process of apoptotic cascades [30, 31]. GSK-3 $\beta$, a critical downstream element of the Akt pathway, participated in physiological and pathological processes such as regulation of glycogen synthesis and disposal, as well as cell death [32]. It is well accepted that glucose utilization is decreased and FFA oxidation is increased in the diabetic heart [33].
This substrate utilization shift has been known to contribute to the pathogenesis of DCM. Thus, activating Akt phosphorylation and inhibiting GSK- $3 \beta$ activity may be considered as cardioprotective actions, as they maintain the physiological growth and functions of the heart and promote cell survival. In the present study, our results indicated that cardiomyocytes exposed to high glucose have strikingly decreased expression levels of Akt and GSK-3 $\beta$ phosphorylation, which is in accordance with the previous report [34]. Interestingly, the beneficial roles exerted by curcumin in high glucoseinduced cardiac injury such as enhancement of Akt and GSK- $3 \beta$ phosphorylation, reduction of gp $91^{\text {phox }}$ and $\mathrm{p} 47^{\text {phox }}$ expression, and regulatory apoptosis-related proteins were negated by the application of LY294002, which indicate that the PI3K/Akt/GSK-3 $\beta$ signalling pathway may be responsible for the inhibition of high glucose-induced cardiac injury by curcumin.

In summary, curcumin exerts cardioprotection against high glucose-induced cardiomyocyte apoptosis, and these effects were shown to possibly be due to efficient prevention of NADPH oxidase-derived oxidative stress and preservation of Akt and GSK-3 $\beta$ phosphorylation in vitro. Therefore, curcumin may be a feasible novel drug for the treatment of DCM.

\section{Conflict of Interests}

The authors declare that there is no conflict of interests regarding the publication of this paper. 


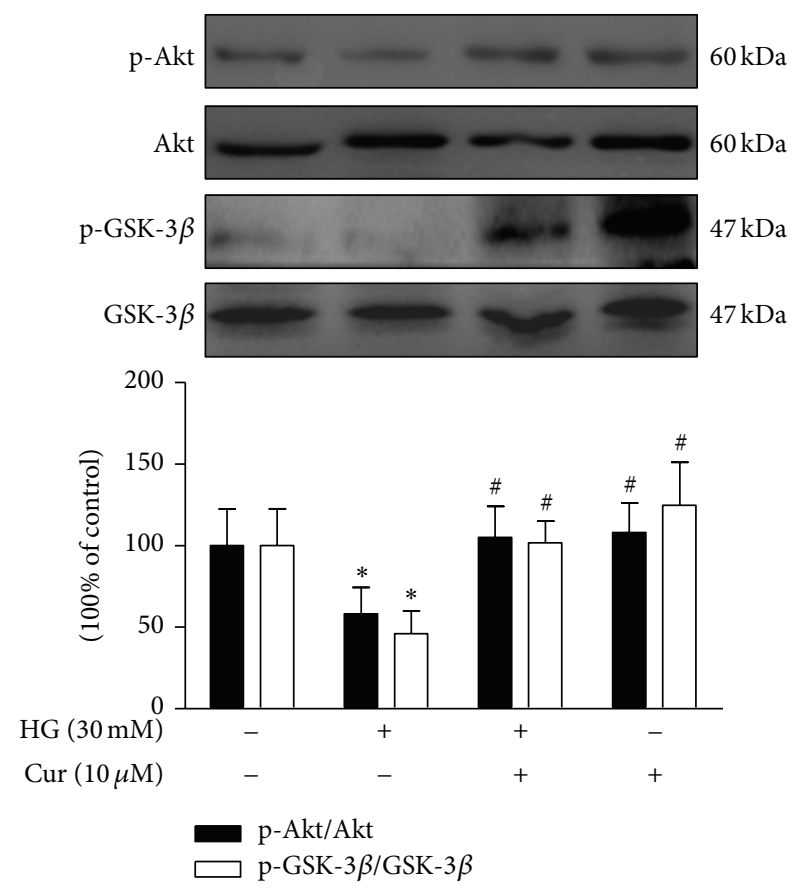

(a)

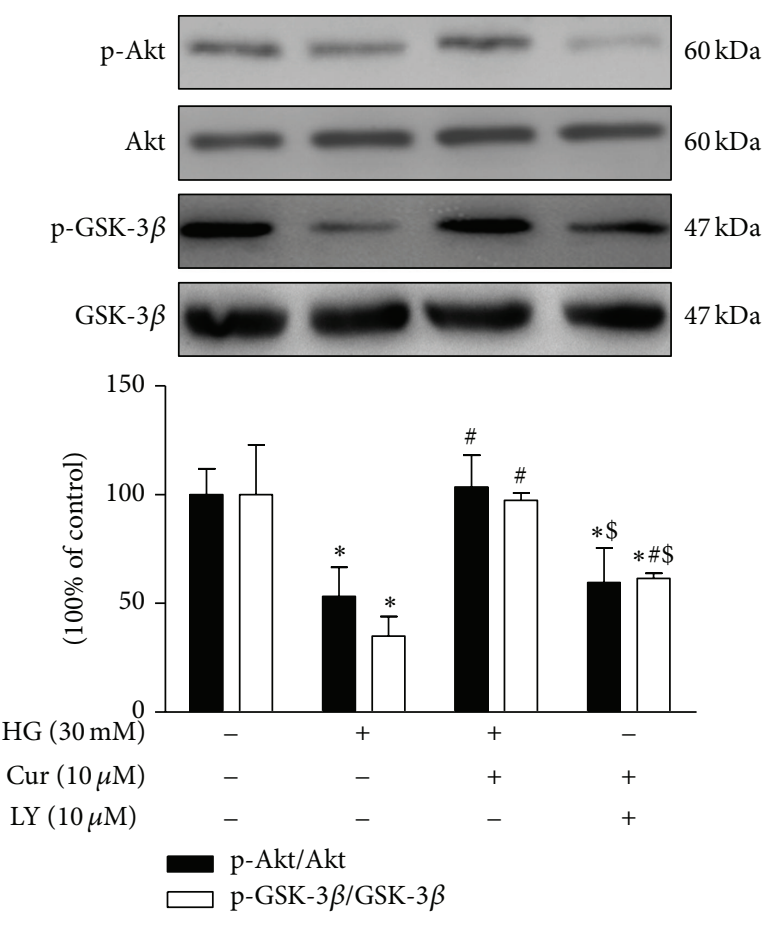

(b)

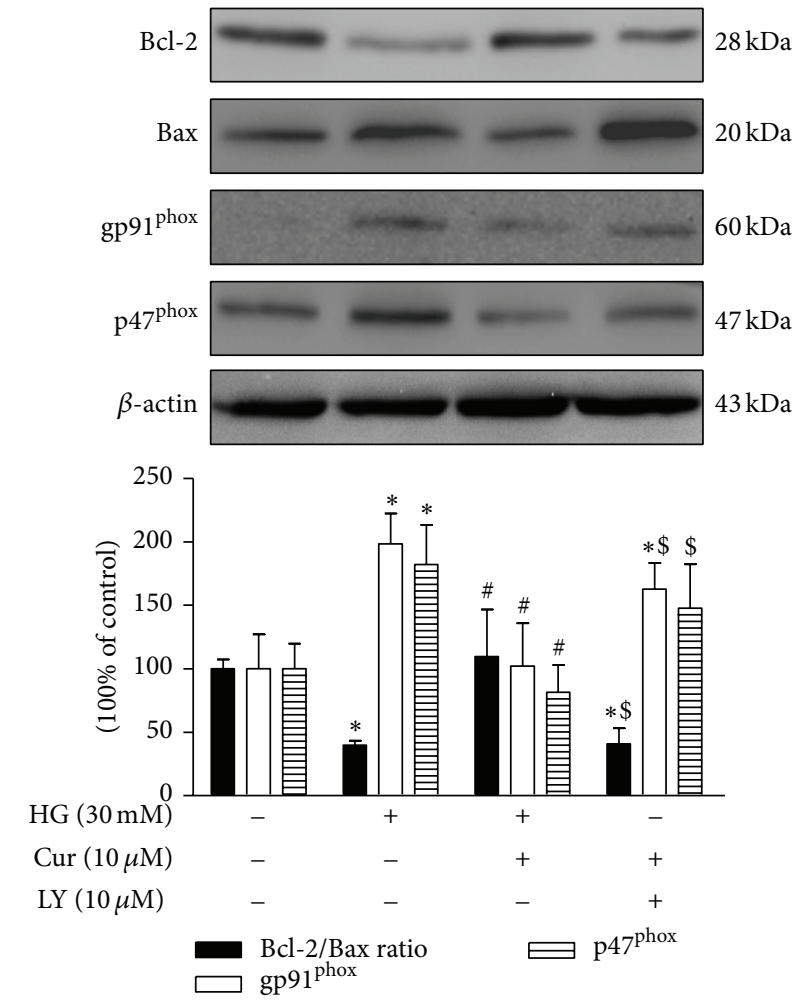

(c)

Figure 6: Curcumin inhibited high glucose-induced apoptosis and oxidative stress via activation of Akt in cardiomyocytes. (a) Curcumin upregulated Akt and GSK-3 $\beta$ phosphorylation levels. (b) Increased Akt and GSK-3 $\beta$ phosphorylation by curcumin were abolished by treatment with LY294002. (c) The effects on the Bcl-2/Bax ratio and the expression of gp $91^{\text {phox }}$ and $447^{\text {phox }}$ by curcumin were blocked by treatment with LY294002. $n=3$. Values are presented as mean \pm SD. ${ }^{*} P<0.05$ versus NG group. ${ }^{\#} P<0.05$ versus HG group. ${ }^{\$} P<0.05$ versus curcumin treatment group. 


\section{Authors' Contribution}

Wei Yu and Wenliang Zha contributed equally to this work.

\section{Acknowledgments}

This work was supported by the following grants: Hubei Province Natural Science Fund Project of outstanding youth project awarded to Dr. Chao Liu (no. 2012FFA005), New Century Excellent Talents Project of Ministry of Education awarded to Dr. Chao Liu (no. NCET-13-0781), the National Natural Science Foundation of China awarded to Dr. Wei Yu (no. 81500296), the Hubei Province Natural Science Fund Project awarded to Dr. Wei Yu (no. 2014CFB382), and the Hubei Province Education Office Fund Project awarded to Dr. Wei Yu (no. D20152802).

\section{References}

[1] L. Guariguata, D. R. Whiting, I. Hambleton, J. Beagley, U. Linnenkamp, and J. E. Shaw, "Global estimates of diabetes prevalence for 2013 and projections for 2035," Diabetes Research and Clinical Practice, vol. 103, no. 2, pp. 137-149, 2014.

[2] W. B. Kannel and D. L. McGee, "Diabetes and cardiovascular disease. The Framingham study," The Journal of the American Medical Association, vol. 241, no. 19, pp. 2035-2038, 1979.

[3] Z. V. Wang and J. A. Hill, "Diabetic cardiomyopathy: catabolism driving metabolism," Circulation, vol. 131, no. 9, pp. 771-773, 2015.

[4] S. Rubler, J. Dlugash, Y. Z. Yuceoglu, T. Kumral, A. W. Branwood, and A. Grishman, "New type of cardiomyopathy associated with diabetic glomerulosclerosis," The American Journal of Cardiology, vol. 30, no. 6, pp. 595-602, 1972.

[5] I. G. Poornima, P. Parikh, and R. P. Shannon, "Diabetic cardiomyopathy: the search for a unifying hypothesis," Circulation Research, vol. 98, no. 5, pp. 596-605, 2006.

[6] A. Frustaci, J. Kajstura, C. Chimenti et al., "Myocardial cell death in human diabetes," Circulation Research, vol. 87, no. 12, pp. 1123-1132, 2000.

[7] C. He, H. Zhu, H. Li, M.-H. Zou, and Z. Xie, "Dissociation of Bcl-2-Beclin1 complex by activated AMPK enhances cardiac autophagy and protects against cardiomyocyte apoptosis in diabetes," Diabetes, vol. 62, no. 4, pp. 1270-1281, 2013.

[8] R. Tarquini, C. Lazzeri, L. Pala, C. M. Rotella, and G. F. Gensini, "The diabetic cardiomyopathy," Acta Diabetologica, vol. 48, no. 3, pp. 173-181, 2011.

[9] J. Kajstura, F. Fiordaliso, A. M. Andreoli et al., "IGF-1 overexpression inhibits the development of diabetic cardiomyopathy and angiotensin II-mediated oxidative stress," Diabetes, vol. 50, no. 6, pp. 1414-1424, 2001.

[10] A. Modesti, I. Bertolozzi, T. Gamberi et al., "Hyperglycemia activates JAK2 signaling pathway in human failing myocytes via angiotensin $I I$-mediated oxidative stress," Diabetes, vol. 54, no. 2, pp. 394-401, 2005.

[11] F. Fiordaliso, R. Bianchi, L. Staszewsky et al., "Antioxidant treatment attenuates hyperglycemia-induced cardiomyocyte death in rats," Journal of Molecular and Cellular Cardiology, vol. 37, no. 5, pp. 959-968, 2004.

[12] J.-M. Zingg, S. T. Hasan, and M. Meydani, "Molecular mechanisms of hypolipidemic effects of curcumin," BioFactors, vol. 39, no. 1, pp. 101-121, 2013.
[13] M. Izem-Meziane, B. Djerdjouri, S. Rimbaud et al., "Catecholamine-induced cardiac mitochondrial dysfunction and mPTP opening: protective effect of curcumin," The American Journal of Physiology-Heart and Circulatory Physiology, vol. 302, no. 23, pp. 665-674, 2012.

[14] Y. Pan, Y. Wang, Y. Zhao et al., "Inhibition of JNK phosphorylation by a novel curcumin analog prevents high glucoseinduced inflammation and apoptosis in cardiomyocytes and the development of diabetic cardiomyopathy," Diabetes, vol. 63, no. 10, pp. 3497-3511, 2014.

[15] W. Yu, J. Wu, F. Cai et al., "Curcumin alleviates diabetic cardiomyopathy in experimental diabetic rats," PLOS ONE, vol. 7, no. 12, Article ID e52013, 2012.

[16] C. W. Younce, M. A. Burmeister, and J. E. Ayala, "Exendin-4 attenuates high glucose-induced cardiomyocyte apoptosis via inhibition of endoplasmic reticulum stress and activation of SERCA2a," American Journal of Physiology-Cell Physiology, vol. 304, no. 6, pp. C508-C518, 2013.

[17] J. Zielonka, M. Hardy, and B. Kalyanaraman, "HPLC study of oxidation products of hydroethidine in chemical and biological systems: ramifications in superoxide measurements," Free Radical Biology and Medicine, vol. 46, no. 3, pp. 329-338, 2009.

[18] J. Li, H. Zhu, E. Shen, L. Wan, J. M. O. Arnold, and T. Peng, "Deficiency of Racl blocks NADPH oxidase activation, inhibits endoplasmic reticulum stress, and reduces myocardial remodeling in a mouse model of type 1 diabetes," Diabetes, vol. 59, no. 8, pp. 2033-2042, 2010.

[19] Y. Zhang, S. A. Babcock, N. Hu, J. R. Maris, H. Wang, and J. Ren, "Mitochondrial aldehyde dehydrogenase (ALDH2) protects against streptozotocin-induced diabetic cardiomyopathy: role of GSK $3 \beta$ and mitochondrial function," BMC Medicine, vol. 10, article 40, 2012.

[20] B. DeBosch, I. Treskov, T. S. Lupu et al., "Akt1 is required for physiological cardiac growth," Circulation, vol. 113, no. 17, pp. 2097-2104, 2006.

[21] Y. Liu, Z. Huang, L. Wang et al., "Sitagliptin alleviated myocardial remodeling of the left ventricle and improved cardiac diastolic dysfunction in diabetic rats," Journal of Pharmacological Sciences, vol. 127, no. 3, pp. 260-274, 2015.

[22] F. Giacco and M. Brownlee, "Oxidative stress and diabetic complications," Circulation Research, vol. 107, no. 9, pp. 10581070, 2010.

[23] W.-W. Kuo, W.-J. Wang, C.-Y. Tsai, C.-L. Way, H.-H. Hsu, and L.-M. Chen, "Diallyl trisufide (DATS) suppresses high glucoseinduced cardiomyocyte apoptosis by inhibiting JNK/NF $\kappa \mathrm{B}$ signaling via attenuating ROS generation," International Journal of Cardiology, vol. 168, no. 1, pp. 270-280, 2013.

[24] K.-H. Tsai, W.-J. Wang, C.-W. Lin et al., "NADPH oxidasederived superoxide anion-induced apoptosis is mediated via the JNK-dependent activation of NF- $\kappa \mathrm{B}$ in cardiomyocytes exposed to high glucose," Journal of Cellular Physiology, vol. 227, no. 4, pp. 1347-1357, 2012.

[25] P. L. Hordijk, "Regulation of NADPH oxidases: the role of Rac proteins," Circulation Research, vol. 98, no. 4, pp. 453-462, 2006.

[26] E. Shen, Y. Li, Y. Li et al., "Racl is required for cardiomyocyte apoptosis during hyperglycemia," Diabetes, vol. 58, no. 10, pp. 2386-2395, 2009.

[27] X. Sun, R.-C. Chen, Z.-H. Yang et al., "Taxifolin prevents diabetic cardiomyopathy in vivo and in vitro by inhibition of oxidative stress and cell apoptosis," Food and Chemical Toxicology, vol. 63, pp. 221-232, 2014. 
[28] R. G. Katare, A. Caporali, A. Oikawa, M. Meloni, C. Emanuel, and P. Madeddu, "Vitamin B1 analog benfotiamine prevents diabetes-induced diastolic dysfunction and heart failure through Akt/Pim-1-mediated survival pathway," Circulation: Heart Failure, vol. 3, no. 2, pp. 294-305, 2010.

[29] Z.-Z. Yang, O. Tschopp, N. Di-Poï et al., "Dosage-dependent effects of Akt1/protein kinase $\mathrm{B} \alpha(\mathrm{PKB} \alpha)$ and $\mathrm{Akt} 3 / \mathrm{PKB} \gamma$ on thymus, skin, and cardiovascular and nervous system development in mice," Molecular and Cellular Biology, vol. 25, no. 23, pp. 10407-10418, 2005.

[30] H. Cho, J. Mu, J. K. Kim et al., "Insulin resistance and a diabetes mellitus-like syndrome in mice lacking the protein kinase Akt2 (PKB $\beta)$," Science, vol. 292, no. 5522, pp. 1728-1731, 2001.

[31] B. DeBosch, N. Sambandam, C. Weinheimer, M. Courtois, and A. J. Muslin, "Akt2 regulates cardiac metabolism and cardiomyocyte survival," Journal of Biological Chemistry, vol. 281, no. 43, pp. 32841-32851, 2006.

[32] Y. Wang, W. Feng, W. Xue et al., "Inactivation of GSK-3 $\beta$ by metallothionein prevents diabetes-related changes in cardiac energy metabolism, inflammation, nitrosative damage, and remodeling," Diabetes, vol. 58, no. 6, pp. 1391-1402, 2009.

[33] P. Razeghi, M. E. Young, T. C. Cockrill, O. H. Frazier, and H. Taegtmeyer, "Downregulation of myocardial myocyte enhancer factor $2 \mathrm{C}$ and myocyte enhancer factor 2C-regulated gene expression in diabetic patients with nonischemic heart failure," Circulation, vol. 106, no. 4, pp. 407-411, 2002.

[34] S. Guo, Q. Yao, Z. Ke, H. Chen, J. Wu, and C. Liu, "Resveratrol attenuates high glucose-induced oxidative stress and cardiomyocyte apoptosis through AMPK," Molecular and Cellular Endocrinology, vol. 412, pp. 85-94, 2015. 


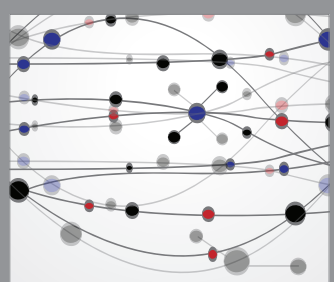

The Scientific World Journal
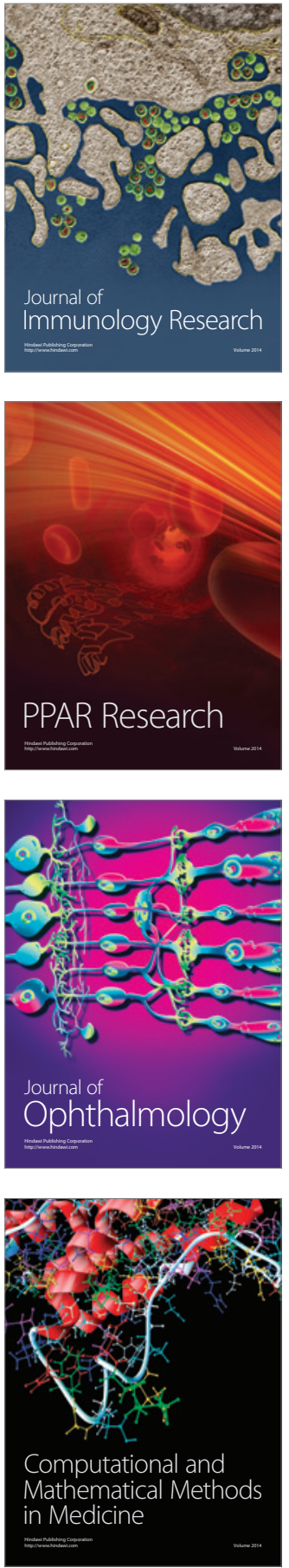

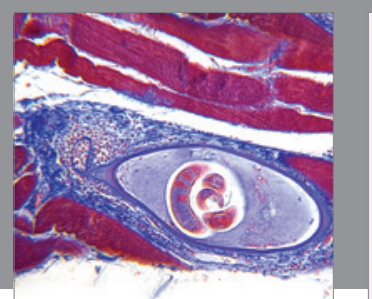

Gastroenterology Research and Practice

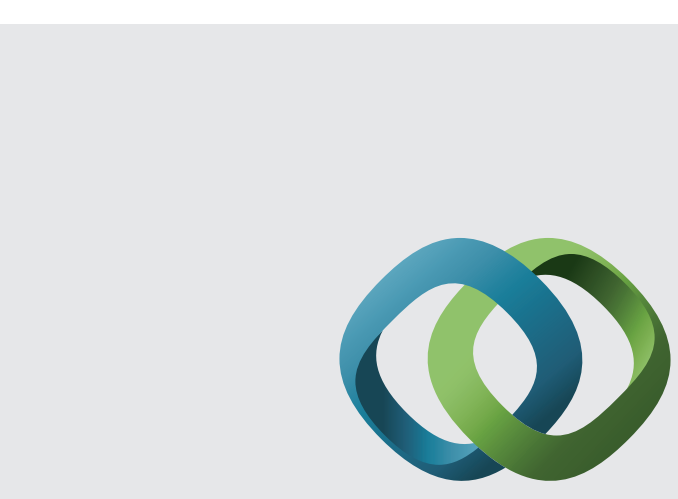

\section{Hindawi}

Submit your manuscripts at

http://www.hindawi.com
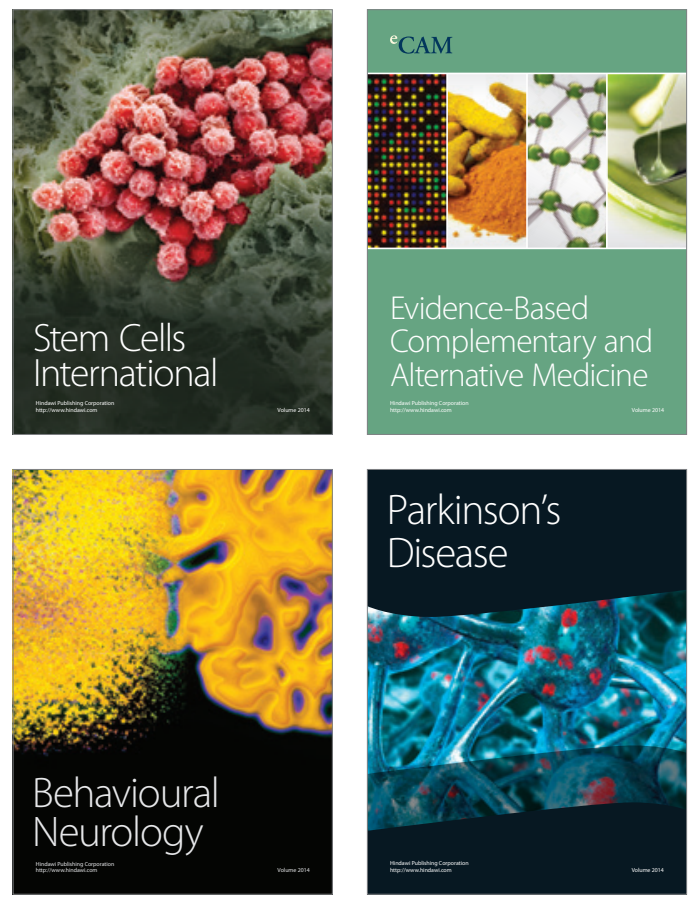
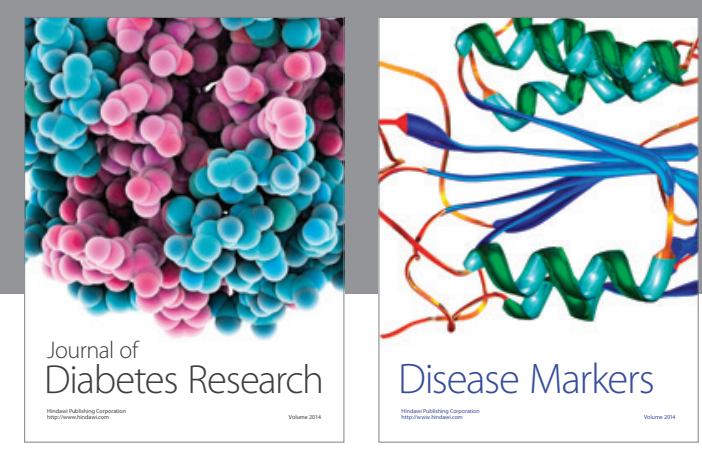

Disease Markers
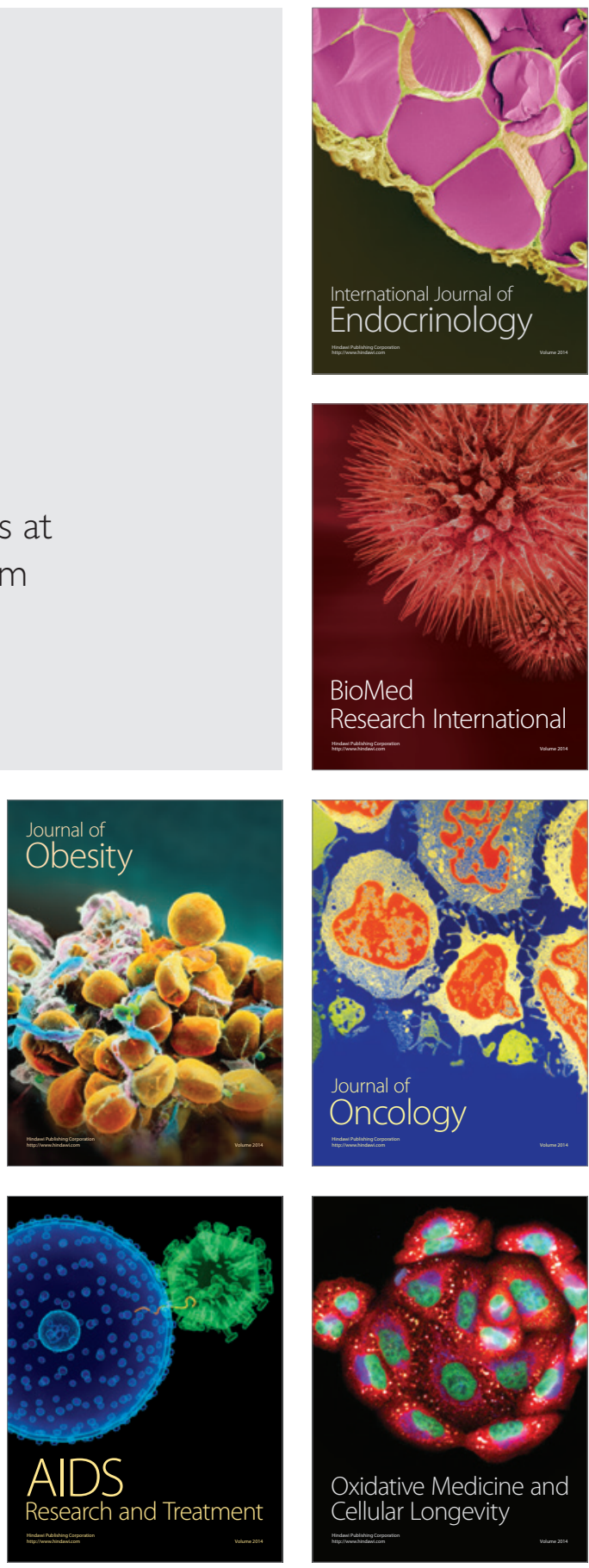